\title{
BIOMASA DE MACRÓFITOS ACUÁTICOS EN LA MARISMA DEL PARQUE NACIONAL DE DOÑANA (SW ESPAÑA): IMPORTANCIA Y FACTORES AMBIENTALES QUE CONTROLAN SU DISTRIBUCIÓN
}

\author{
C. Duarte, ${ }^{1}$ C. Montes,' S. Agustí, ${ }^{1}$ P. Martino, ${ }^{2}$ M. Bernués ${ }^{Z}$ y J. Kalff \\ 1. Centro de Estudios Avanzados. CSIC. Camino de Santa Bárbara s/n. Blanes, Girona. Spain. \\ 2. Departamento de Ecología, Facultad de Ciencias, Universidad Autónoma de Madrid. Cantoblanco, 28049 Ma- \\ drid. Spain. \\ 3. Departament of Biology, McGill University. 1205 Av. Docteur Penfield, Montreal, P.Q. Canada H3A 1 B1.
}

Palabras clave: Doñana salt-marsh, macrophytes, biomass patterns, environmental factors, Spain.

\author{
ABSTRACT
}

\section{AQUATIC MACROPHYTES BIOMASS OF THE DOÑANA NATIONAL PARK MARSH (SW SPAIN): IMPORTANCE AND ENVIRONMENTAL FACTORS CONTROLLING DISTRIBUTION PATTERNS}

The biomass and importance of aquatic macrophytes for the metabolism of the Doñana National Park marsh (SW Spain) has been studied. Average submerged biomass (591 $\mathrm{g}$ fresh $\mathrm{wt} \mathrm{\textrm {m } ^ { - 2 }}$ ) was greater than the average biomass of emergent $\left(213 \mathrm{~g}\right.$ fresh $\left.\mathrm{wt}^{-2}\right)$, and floating $\left(69 \mathrm{~g}\right.$ fresh $\left.\mathrm{wt} \mathrm{m}^{-2}\right)$ macrophytes. The high biomass of submerged macrophytes resulted in an important contribution of these plants to the spatial and temporal variations in the metabolism (measured as changes in $\mathrm{pH}$ and oxygen concentration) of the aquatic compartment of the marsh. While submerged macrophytes contributed to marsh metabolism through photosynthesis and respiration, emergent and floating macrophytes only contnbuted through respiration.

The species composition of the submerged macrophyte communities was largely controlled by water salinity (1-45 mS). Submerged biomass, however, was unaffected by salinity and was controlled largely by undenvater light levels, which are a function of water transparency and depth. Livestock trampling and flamingos treading in the brackish water of marshes and the Louisiana red swamp crayfish feeding practices in freshwater areas significantly reduced submerged biomass both by a direct influence through physical damage, and indirectly by increasing the area of bare sediments exposed to wave-induced resuspension, increasing water thereby turbidity. We hypothesize that increased water turbidity and the associated reduction in submerged cover propagate through a positive feed-back mechanism.

Our results show that the submerged macrophyte populations of the Doñana National Park marsh is a major contributor to marsh primary production. This population appears to be extremely fragile, and is particularly sensitive to any action that influence water turbidity. Consequently, management action that may impinge upon water turbidity will have a considerable impact upon the metabolism of the aquatic compartment of the marsh.

\section{INTRODUCCIÓN}

La marisma del Parque Nacional de Doiiana es conocida internacionalmente por ser uno de los enclaves más importantes para la invernada, reproducción y descanso de muchas aves acuáticas europeas. Dado que la biomasa media anual de Limnetica, 6: 1-12 (1990)

(C) Asociación Española de Limnología, Madrid, Spain su fitoplancton es muy baja (MONTES et al., 1982a), la producción del sistema, considerada muy alta en lo concerniente a aves acuáticas (AMAT, 1980), invertebrados (MonTES et al., 1982b) y peces (HERNANDO, 1978) debe basarse en la producción de macrófitos acuáticos y algas epifíticas asociadas. Esta elevada producción está de acuerdo con el alto cociente superficie de la cubetaívolumen de agua y permite suponer que los 
tapices o praderas de macrófitos sumergidos, flotantes y helófitos controlan numerosos procesos físicos, metabólicos y ecológicos (CARPENTER \& LodGe, 1986; Symoens, 1988). En este contexto, el estudio limnológico de las comunidades de macrófitos acuáticos adquiere un papel fundamental en el desarrollo de planes de gestión de la zona.

La información sobre la vegetación acuática del Parque Nacional de Doñana es escasa (Bernues $\&$ MonTES, en prensa) y se refiere, principalmente, a aspectos corológicos y fitosociológicos (ver Rivas Martínez et al., 1980). Tan sólo González BERNÁldeZ et al. (1977a,b), TORRES et al. (1978), Allier \& Bresset (1978) y Allier et al. (1978) examinan algunos de los factores que controlan la distribución espacial de algunas especies de plantas acuáticas de la marisma dulce del Martinazo.

En este artículo se presentan los primeros datos sobre biomasa e importancia ecológica de la vegetación acuática de la marisma del Parque Nacional de Doñana, con especial referencia a los macrófitos sumergidos. Asimismo, se realiza un análisis preliminar de los factores ambientales que determinan su biomasa.

La importancia de los macrófitos en la producción primaria y el metabolismo de los ecosistemas acuáticos de la marisma se estableció mediante el estudio de sus componentes espacial (variación entre estaciones) y temporal (variaciones diarias durante un ciclo estacional). Esta importancia se cuantificó indirectamente a través de los cambios de concentración de oxígeno disuelto y de $\mathrm{pH}$ de las aguas (GOULDER, 1970). Para la caracterización general del cuadro de interrelaciones entre la biomasa de macrófitos y factores ambientales se estudió: 1) la profundidad, que por un lado determina el espacio disponible para el crecimiento de los macrófitos sumergidos (Lind \& CotTAM, 1969), y por otro es un componente importante de la disipación de la luz en la columna de agua; 2 ) la salinidad, que parece tener una influencia negativa en el crecimiento y la producción de macrófitos sumergidos (DUARTE et al., 1986); y 3) la exposición relativa al oleaje, ya que éste puede tener una incidencia importante tanto por su acción mecánica sobre las plantas (Jupp \& Spence, 1977; Duarte \& KALFF, 1988) como por su influencia en la resuspensión de sedimentos, que dificulta la penetración de la luz.

\section{MATERIAL Y MÉTODOS}

\section{Área de estudio}

La marisma del Parque Nacional Doñana forma parte de una de las dos grandes unidades ambientales que conforman la llanura de la desembocadura del río Guadalquivir (fig. 1). Constituye un delta interno de gran extensión que en la actualidad se encuentra en un avanzado estadio de su evolución geomorfológica, por lo que presenta un régimen hídrico, bajo un clima mediterráneo, con un dinamismo estacional (ciclo inundación-desecación) y no mareal (Tenajas, 1984).

Por su aislamiento respecto al estuario del Guadalquivir, la cubeta arcillosa de la marisma del Parque se presenta como un gran receptáculo (27 000 ha) de origen fluvio-marino donde, según el balance anual precipitación-temperatura, el período de almacenamiento de agua dulce es de 6 meses (octubre-marzo), el de retención, de 2 meses (marzo-mayo), y, a partir del mes de mayo, el balance aportes-evaporación se hace negativo y la marisma va perdiendo gradualmente agua hasta su completa desecación, a partir de julio. Las mareas sólo tienen importancia en áreas muy localizadas del Parque. En este contexto, la mayoría de los parámetros físico-químicos de la columna de agua presentan un marcado dinamismo estacional. Espacialmente, durante el período de máxima inundación existe una cierta homogeneidad ambiental. Ésta, se va rompiendo a medida que aumenta la tasa de evaporación; pequeñas variaciones de altitud (centímetros) controlan la duración de la inundación y, por tanto, la hidroquímica de sus aguas (MONTES et al., 1982a). Las aguas, de naturaleza cloruradosódisas y con una profundidad media de 20 a $40 \mathrm{~cm}$, son básicamente de origen meteórico y fluvial (arroyo de la Rocina). La variación estacional de la salinidad es muy marcada (mixo-oligohalinas a hiperhalinas sensu Venice System, 1958) y controlada espacialmente por pequeñas diferencias de profundidad (MONTES et al., $1982 \mathrm{a}, \mathrm{c})$.

Morfológicamente, y bajo una perspectiva limnológica, en la marisma de Doñana aparecen distintas formaciones según la duración de la inundación: lucios (cubetas más o menos redondeadas que mantienen agua durante gran parte del ciclo hidrológico anual), caños (antiguos canales ma- 


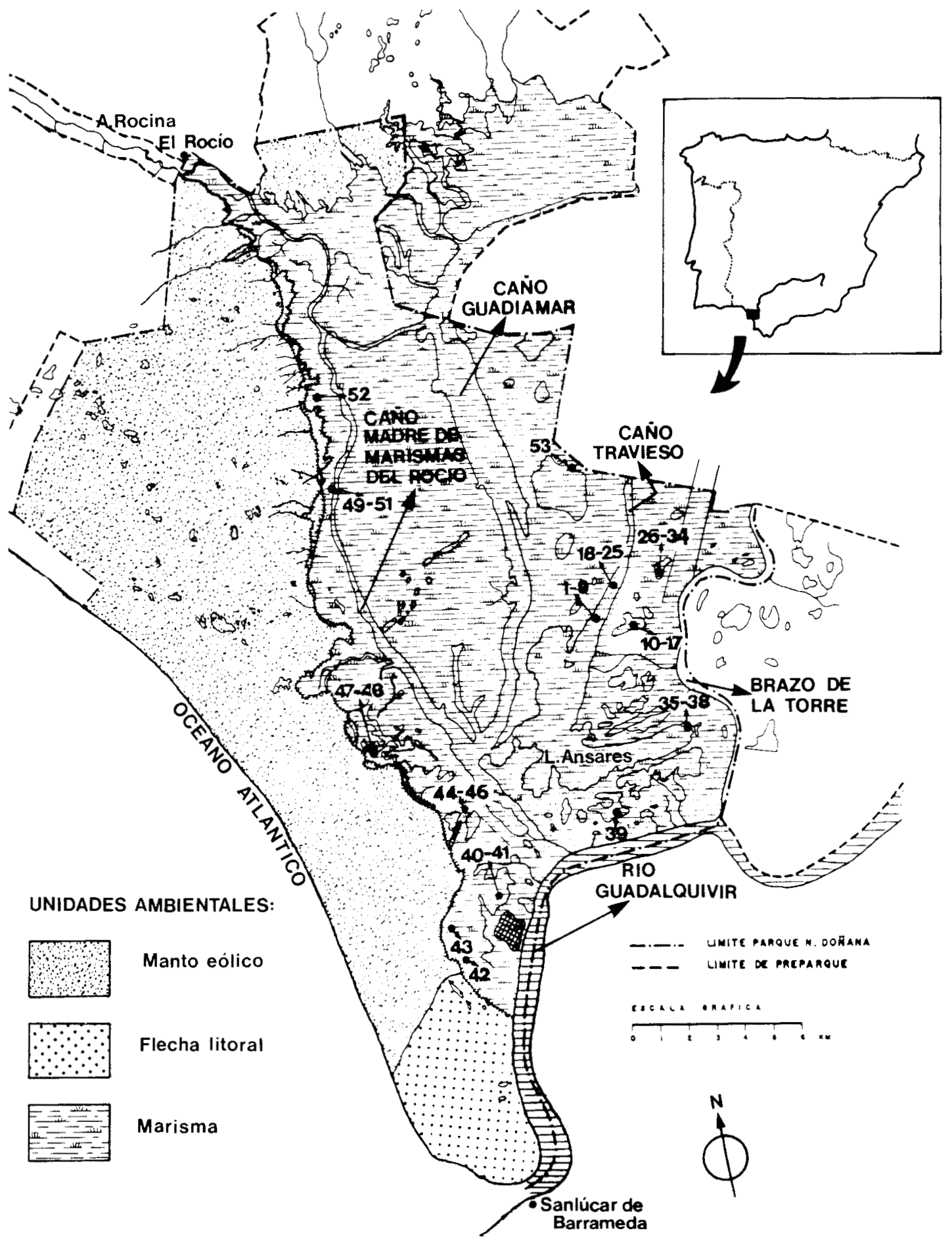

Figura 1.- Distribución en la marisma del Parque Nacional de Doñana de las estaciones de muestreo para el estudio de la bitomasa de macrófitos acuáticos.

Map of the Doñana National Park marshes showing sampling stations. 
reales, hoy en día colmatados pero que mantienen agua durante gran parte del año) y marisma inundable entre lucios y caños, con aguas más o menos profundas y más o menos temporales.

La vegetación de la marisma de Doñana ha sido descrita fitosociológicamente por RIVAs-MARTíneZ et al. (1980). Scirpus littoralis Schr. y Scirpus $m a$ ritimus L. son las especies de helófitos más abundantes, Ranunculus baudotii Godr. desarrolla densos tapices en la superficie del agua y Ruppia drepanensis (Tin.) y Chara galioides D.C. cubren gran parte de sus fondos. Esta vegetación es consumida cada invierno y primavera por mamíferos herbívoros, principalmente especies domésticas (vacas, caballos y ovejas), y aves acuáticas (Anas strepera, Anas crecca, Anas acuta, Aythya ferina, Anas penelope, Fulica atra).

\section{Muestreos y métodos de análisis}

Dentro del área de estudio, que abarca la marisma comprendida dentro de los límites del Parque Nacional de Doñana, se seleccionaron 53 estaciones de muestreo distribuidas de modo que cubrieran el mayor rango de condiciones ambientales posibles (fig. 1). Las estaciones fueron muestreadas entre el 25 de abril y el 5 de mayo de 1987, coincidiendo con el período de máxima inundación y producción vegetal de la zona (marzomayo; MonTEs et al., 1982b).

En cada estación de muestreo se distribuyeron al azar tres cuadrados $\left(0,16 \mathrm{~m}^{2}\right)$ y se recogió la biomasa epigea de los macrófitos acuáticos comprendidos en cada uno. Las plantas se identificaron en el campo y se agruparon en tres grupos funcionales: macrófitos acuáticos sumergidos, flotantes y helófitos. Se estimó el peso fresco de cada grupo mediante dinamómetros tras centrifugar las plantas en redes para eliminar el exceso de agua (CANFIELD \& HOYER, 1988). En los análisis estadísticos se utilizó el valor medio de las tres unidades de muestreo recogidas en cada estación.

Al mismo tiempo que se realizaban las estimas de biomasa en cada estación de muestreo se midió la temperatura del aire y del agua (termistor Instran-10), pH (indicadores Merck), conductividad eléctrica (conductivímetro Instran-10), oxígeno disuelto (oxímetro Simplair-L), transparencia (disco de Secchi negro de $8 \mathrm{~cm}$, Davies-Colley,
1988) y la profundidad del agua en cada cuadrado. El efecto del ganado se estimó empleando una escala semicuantitativa: $0=\sin$ ganado, $1=$ baja (menos de 13 pisadas $/ \mathrm{m}^{2}$ ), $2=$ media (entre $13 \mathrm{y}$ 50 pisadas $/ \mathrm{m}^{2}$ ) y $3=$ alta (más de 50 pisadas $/ \mathrm{m}^{2}$ ).

En el laboratorio se calculó mediante un mapa 1:5000 la exposición relativa a las olas, evaluada como la superficie de agua visible desde cada estación de muestreo (DuARTE \& KALFF, 1988). Debido a la resolución del mapa, a los valores menores de $625 \mathrm{~m}^{2}$ se les asignó el valor $\mathrm{O}$.

Los ciclos diarios de algunos parámetros físicos y químicos del agua (temperatura y oxígeno disuelto) se realizaron en una estación ( $\mathrm{n}^{\circ} 3$, fig. 1.) en tres períodos del ciclo anual de inundación 1985-86 (llenado, 24/I/86; retención, 7/V/86; vaciado, 28/V/86).

Las relaciones entre los datos obtenidos se cuantificaron mediante análisis de correlación y regresión lineal. En aquellos casos en los que fue necesario, los datos fueron transformados adecuadamente.

\section{RESULTADOS}

\section{Características del medio relacionadas con la distribución de macrófitos acuáticos}

Las aguas de la marisma son muy someras (tabla 1), lo que se traduce en una elevada razón superficie de la cubeta/volumen de agua, que determina un acoplamiento atmósfera-agua muy estrecho.

La turbiedad y transparencia del agua muestran una gran variabilidad horizontal en la marisma de Doñana (tabla 1; 0,6 a 330 unidades nefelométricas). Los valores de transparencia vienen condicionados por la cantidad de sedimentos finos (arcillas y limos) resuspendidos en la columna de agua, ya que la biomasa del fitoplancton durante la mayor parte del ciclo anual es muy baja (MoNTES et al., 1982a). Las zonas menos profundas y más expuestas a la acción del oleaje $(10-20 \mathrm{~cm}$ de amplitud) son las que mantienen durante otoño e invierno las aguas menos transparentes (valores del disco de Secchi entre 5 y $7 \mathrm{~cm}$ ). En este contexto, las zonas de marisma protegidas del oleaje por la vegetación de almajos (Arthrocnemum spp) 
Tabla 1.- Media (X), desviación típica (S). coeficiente de variación $(\mathrm{CV})$ y rango de los parámetros físico químicos y biológicos medidos en las aguas de la marisma de Doñana. Todas las biomasas se expresan en $\mathrm{g}$ (peso fresco) $\mathrm{m}^{-2} ; \mathrm{n}=53$; la transparencia se expresa en porcentaje de la visibilidad del disco de Secchi en una columna de agua.

Mean, standard deviation, coefficient of variation and range of the physicochemical and biological parameters analyzed in the waters of Doñana marsh. All the biomass values are expressed as $\mathrm{g}$ (fresh weight) $\mathrm{m}^{-2} ; \mathrm{n}=53$; water transparence is expressed as percentage of the visibility of the Secchi disc in the water column

\begin{tabular}{|c|c|c|c|c|}
\hline Variable & $\bar{x}$ & $S$ & $C V(\%)$ & Rango \\
\hline Temperatura agua ("C) & 24,8 & 2,7 & 10,9 & $19,5-30,0$ \\
\hline Profundidad $(\mathrm{cm})$ & 6,3 & 4,3 & 16,3 & $7,2-80,4$ \\
\hline Transparencia (\%) & 64,0 & 37,0 & 58,0 & $0,0-100$ \\
\hline Exposición al oleaje (ha) & 4,2 & 10,5 & 254,2 & $0-50,1$ \\
\hline Conductividad ( $\mathrm{mS} \mathrm{cm-')}$ & 15,2 & 7,1 & 45,8 & $0,9-47,3$ \\
\hline Oxígeno $\left(\mathrm{mg} \mathrm{l}^{-1}\right)$ & 11,8 & 3,8 & 32,2 & $1,2-16,1$ \\
\hline $\mathrm{pH}$ & 8,5 & 0,88 & 10,4 & $6,5-10,0$ \\
\hline $\begin{array}{l}\text { Biomasa } \\
\text { macrófitos sumergidos } \\
\text { Biomasa }\end{array}$ & 591,1 & 881,8 & 149,2 & $0-4843$ \\
\hline macrófitos flotantes & 69,3 & 278,6 & 402 & $0-1586$ \\
\hline Biomasa helófitos & 213,2 & 520,8 & 244,3 & $0-2218$ \\
\hline
\end{tabular}

Tabla 2.- Distribución, según dos clases de conductividad, de las especies más abundantes de macrófitos acuáticos presentes en las estaciones muestreadas.

Distribution of the most abundant aquatic macrophyte species according to two classes of conductivity.

\begin{tabular}{lll}
\hline & \multicolumn{2}{c}{ Conductividad } \\
\cline { 2 - 3 } Macrófitos & $47.10 \mathrm{mS}$ & $2.1 \mathrm{mS}$ \\
\hline
\end{tabular}

\begin{tabular}{|c|c|c|}
\hline \multicolumn{3}{|l|}{ Sumergidos } \\
\hline Chara galioides D.C. & + & \\
\hline Ruppia drepanensis (Tin). & + & \\
\hline Althenia orientalis (Tzv.) Mur. \& Tal. & + & \\
\hline Potamogeton pectinatus L. & + & \\
\hline Zannichellia obtusifolia Tal. Mur \& Sm. & + & \\
\hline Myriophyllum alterniflorum D.C. & . & \\
\hline \multicolumn{3}{|l|}{ Flotantes } \\
\hline Ranunculus baudotii Godron. & + & \\
\hline Lemna gibba $\mathrm{L}$. & . & \\
\hline \multicolumn{3}{|l|}{ Helófitos } \\
\hline \multicolumn{3}{|l|}{ Scirpus maritimus L. } \\
\hline Scirpus maritimus L. var. maritimus & . & + \\
\hline Scirpus littoralis Schrader & + & . \\
\hline Eleocharis palustris (L.) & & + \\
\hline
\end{tabular}

mantienen aguas más transparentes que los lucios o caños con grandes espacios de agua libre. Este patrón espacial queda modificado durante la primavera por el desarrollo de un tapiz de macrófitos sumergidos que estabiliza las capas superficiales de la cubeta arcillosa.

Durante el período de máxima inundación se observa un gradiente de salinidad, fundamentalmente de este a oeste, atribuible a la descarga natural de las aguas subterráneas, muy poco mineralizadas, del acuífero libre que bordea la marisma a lo largo de toda la zona de ecotono de la Vera y Retuerta (Llamas $e t$ al., 1987), y al arroyo de la Rocina, que introduce importantes volúmenes de agua dulce por el NE (fig. 1), de forma discontinua y torrencial. De esta forma, las muestras tomadas en la zona $\mathrm{W}$ de la marisma (estaciones 1-40) muestran valores de conductividad que oscilan entre 12,2 y $30 \mathrm{mS}$, mientras que las de la zona $\mathrm{E}$ (estaciones 42-52) oscilan entre $11 \mathrm{mS}$, para el extremo meridional del ecotono, y $0,9 \mathrm{mS}$, en las proximidades de la desembocadura del arroyo de la Rocina.

\section{Importancia de las comunidades de macrófitos acuáticos en el metabolismo de la marisma}

La riqueza de especies de las comunidades de macrófitos acuáticos de la marisma (tabla 2) es bastante baja en comparación con los sistemas acuáticos situados sobre el manto eólico (RIVAS Martínez et al., 1980). Esta pobreza de especies refleja el caracter forzado del ambiente de la marisma, donde las marcadas fluctuaciones anuales e interanuales del medio condicionan los valores extremos de algunas variables que controlan la composición específica de las comunidades de macrófitos acuáticos (permanencia de las aguas, salinidad, turbiedad). En particular, nuestros datos apuntan a la salinidad como un factor de gran importancia para explicar diferencias en la presencia de distintas especies (tabla 2).

A pesar de la hostilidad del medio, la biomasa de macrófitos acuáticos sumergidos es considerable $\left(\right.$ media $=591 \mathrm{~g}$ peso fresco $\mathrm{m}^{-2}$, tabla 1$)$ en comparación con los valores registrados para la zona templada (WESTLAKE, 1965), especialmente si se tiene en cuenta el poco espacio disponible para su crecimiento (aguas poco profundas). La biomasa media de macrófitos flotantes y helófitos 
es bastante inferior, incluso si se calcula sólo en las estaciones donde se encontraban presentes. Sin embargo, tanto los helófitos como los macrófitos flotantes pueden alcanzar biomasas considerables (2219 y $1569 \mathrm{~g}$ peso fresco $\mathrm{m}^{-2}$, respectivamente) en ambientes favorables, es decir, en canales o depresiones poco profundas y resguardadas de los vientos para los macrófitos flotantes, y la marisma más dulce y menos profunda para los helófitos.

El metabolismo de la marisma se refleja claramente en la distribución de los valores de oxígeno disuelto y de $\mathrm{pH}$. La actividad fotosintética en este tipo de aguas provoca un aumento en los valores de $\mathrm{pH}$ y $\mathrm{O}_{2}$, mientras que la respiración tiene un efecto opuesto (WeTZEL, 1983). La alta relación que existe entre ambas variables $(r=0,86$, P a 0,001; fig. 2) sólo se explica si su variación conjunta refleja el mismo fenómeno, que, dado el signo positivo de la correlación, parece ser el metabolismo de la comunidad acuática. El hecho de que estas dos variables se comporten en la marisma como un índice metabólico se debe al bajo volumen de agua por unidad de superficie y a la elevada producción biológica de la zona.

Con objeto de estudiar las relaciones entre los valores de biomasa de los macrófitos acuáticos (variable dependiente) y los de los dos indicadores del metabolismo de la marisma $\left(\mathrm{O}_{2} \mathrm{y} \mathrm{pH}\right.$, variables independientes) se realizaron análisis de regresión múltiple paso a paso (DAMONT \& HARvEY, 1987). Debido a los marcados ciclos diarios

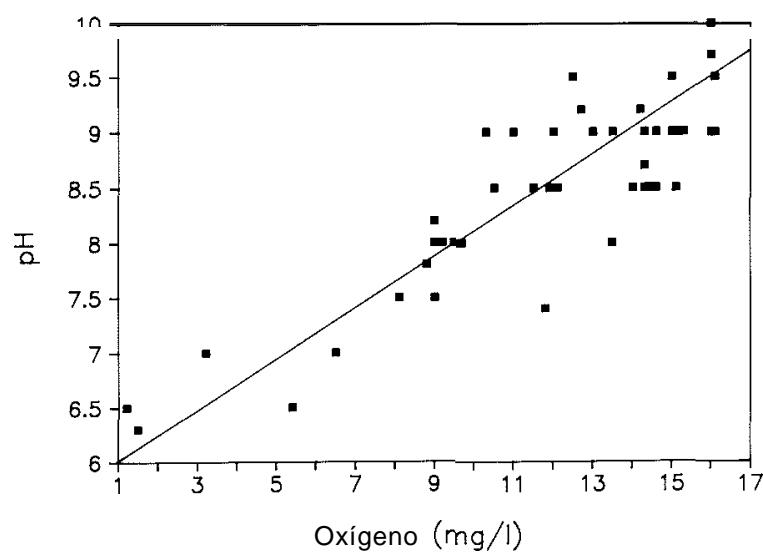

Figura 2.- Relación entre el pH y la concentración de oxígeno disuelto en las aguas de la marisma.

The relationship between $\mathrm{pH}$ and dissolved $\mathrm{O}$, concentration in the marsh waters. (fig. 3) que experimentan estas variables, fue necesario transformar los datos a su raíz cuadrada para ajustar el análisis a las hipótesis implícitas en el modelo de regresión lineal empleado. La tabla 3 presenta algunos parámetros resultantes de los análisis.

La relación positiva entre la biomasa de macrófitos sumergidos y los índices metabólicos (tabla 3) refleja su importancia en la producción primaria de la marisma, mientras que donde hay macrófitos flotantes el agua que queda debajo se empobrece en oxígeno tanto más cuanto mayor es la biomasa de éstos; la biomasa de macrófitos emergentes no se relaciona de forma significativa con los índices metabólicos, ya que su intercambio de gases es principalmente aéreo.

La proporción de la varianza de los índices metabólicos que se explica por las variaciones en la biomasa de macrófitos sumergidos, emergentes y flotantes (tabla 3), representa la contribución relativa de estos grupos a los procesos metabólicos de la marisma. Estas proporciones demuestran que la contribución de los macrófitos sumergidos (fotosíntesis) es sensiblemente más importante que la de los macrófitos flotantes (respiración).

Las variaciones en los valores de $\mathrm{O}_{2}$ y $\mathrm{pH}$ que no se explican por la biomasa de macrófitos acuáticos pueden deberse a la incidencia en el metabolismo de la marisma de otros elementos de la comunidad (por ejemplo, comunidades de microorganismo , bentos, meiobentos, plancton, necton, demanda química de oxígeno, etc.) y a la influencia de factores físicos, como el oleaje o la profundidad.

La estrecha relación que existe entre la biomasa de macrófitos sumergidos y el metabolismo de la marisma también se pone de manifiesto en el examen de los pulsos diarios de $\mathrm{O}_{2}$ disuelto registrados en tres períodos de su ciclo estacional (fig. 3). La variación diaria de la concentración de oxígeno refleja la evolución del metabolismo de la marisma a lo largo del ciclo hidrológico. Durante el período de llenado (aguas relativamente profundas desprovistas de cubierta vegetal sumergida, fig. 3d) los ciclos son atribuibles a procesos físicos (temperatura, difusión, fig. 3a). El grado de control de los macrófitos sumergidos sobre el ritmo metabólico de la marisma se acentúa a medida que crece el tapiz vegetal (fig. $3 b, c)$, que fuerza oscilaciones mucho más marcadas del oxí- 

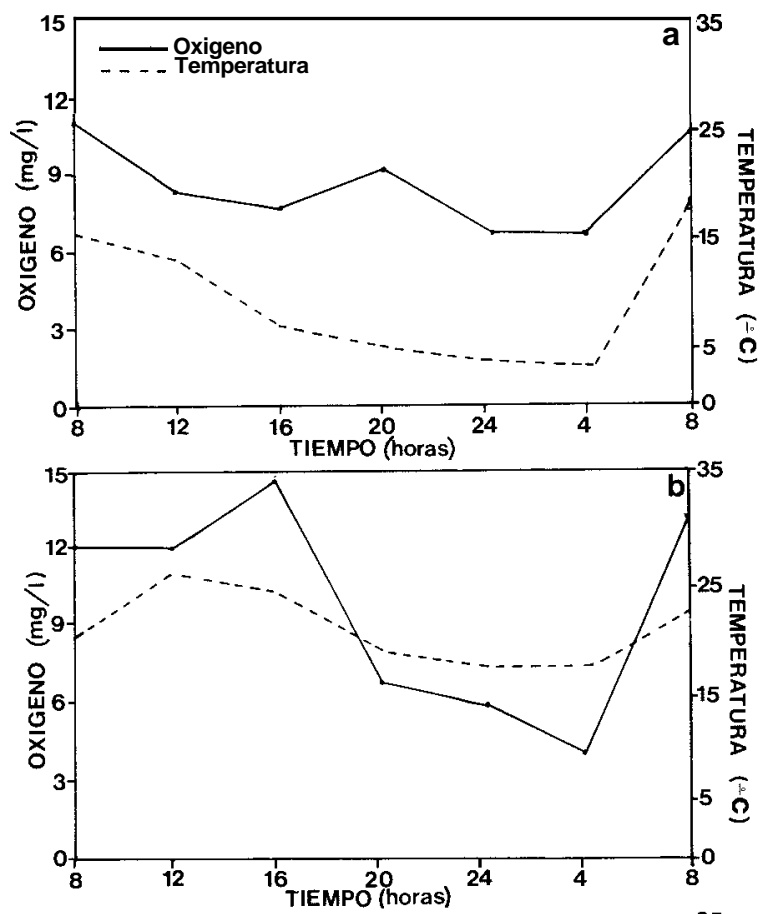

Tabla 3.- Resultados de los análisis de la variación de la biomasa de macrófitos acuáticos en función de algunos indicadores metabólicos ( $\mathrm{pH}$ y oxígeno). Para cada variable se da el coeficiente $b$, la proporción de la variación de la variable dependiente que queda explicada $\left(\mathrm{R}^{\mathrm{Z}}\right)$ y la probabilidad de que $\mathrm{b} \neq 0(\mathrm{P}) . \mathrm{NS}=$ no significativo.

Biomass variation of the aquatic macrophytes according to $\mathrm{pH}$ and oxygen. The $b$ coefficient, percentage of variation of the explained dependent variable $\left(\mathrm{R}^{\mathrm{Z}}\right)$ and the probability for $\mathrm{b} \neq 0(\mathrm{P})$ are given. NS = unsignificant.

\begin{tabular}{lcccccc}
\hline & \multicolumn{3}{c}{$p H$} & \multicolumn{3}{c}{$\boldsymbol{O}_{2}$} \\
\cline { 2 - 7 } Macrófitos & $b$ & $\boldsymbol{R}^{2}$ & $\boldsymbol{P}$ & $\boldsymbol{b}$ & $\boldsymbol{R}^{2}$ & $\boldsymbol{P}$ \\
\hline Sumergidos & 0,023 & $\mathbf{0 , 2 9}$ & 0,001 & $\mathbf{0 , 0 7}$ & $\mathbf{0 , 1 8}$ & 0,001 \\
Flotantes & 0,002 & $\mathbf{0 , 0 3}$ & NS & $-0,004$ & $\mathbf{0 , 0 9}$ & $\mathbf{0 , 0 1}$ \\
Helófitos & 0,000 & $\mathbf{0 , 0 0}$ & NS & 0,000 & $\mathbf{0 , 0 0}$ & NS \\
\hline
\end{tabular}

geno disuelto. Sin embargo, la influencia de los macrófitos sobre las oscilaciones de oxígeno disuelto no son simplemente una función de su biomasa (que influye en la producción de oxígeno) sino que dependen también de la altura de la co lumna de agua (fig. 3d), que controla el grado de dilución del oxígeno producido. Por ello, la intensidad de los ciclos diarios no es máxima cuando la producción o biomasa de macrófitos sumergidos es máxima (período de retención hídrica, fig. 3b, d) sino cuando la razón biomasa/volumen de agua es mayor (período de vaciado, fig. 3c, d).

\section{El papel de los factores ambientales en las variaciones de la biomasa de macrófitos}

La impresión de uniformidad que la marisma ofrece al observador contrasta con la gran variación que se registra en los valores de biomasa de macrófitos acuáticos $(\mathrm{CV}=149-402 \%$, tabla 2$)$, que indica que la heterogeneidad ambiental debe ser mayor de lo que inicialmente se percibe.

Para evaluar la influencia de una serie de factores del medio, exposición al oleaje, transparencia, profundidad y conductividad (variables independientes) sobre la variación de la biomasa de macrófitos sumergidos (variable dependiente), se realizó un análisis de regresión múltiple paso a paso. Este análisis no se intentó para los macrófitos flotantes y helófitos debido al escaso número de muestras en las que se obtuvieron registros ( $\mathrm{n}=5$ y 13 , respectivamente). Además, el análisis de la biomasa de macrófitos sumergidos se limitó a aquellas muestras en las que los valores de bio- 
masa de macrófitos flotantes eran inferiores a $\mathbf{5 0 0}$ $\mathrm{g}$ (peso fresco $\mathrm{m}^{-2}$ ), que es el valor crítico registrado a partir del cual el sombreado de las hojas flotantes de Ranunculus baudotii impide el desarrollo de cualquier población de macrófito sumergido. En estos lugares el denso tapiz de macrófitos flotantes que cubre la superficie del agua condiciona fondos con poco oxígeno y reductores (1-3 $\left.\mathrm{mg} \mathrm{O}_{2} / 1\right)$. Esta interacción negativa, por competencia por la luz, entre especies acuáticas de $R a$ nunculus y macrófitos sumergidos ha sido descrita con anterioridad y puede verse aumentada por la producción de sustancias tóxicas que limitan el crecimiento de ambos grupos (Grillas \& DuNCAN, 1986).

El modelo de regresión múltiple que se obtuvo apunta a la transparencia y la profundidad del agua como las variables que explican un mayor porcentaje de la varianza de la biomasa de macrófitos sumergidos (coeficiente de determinación $\left.\mathrm{R}^{2}=0,5\right)$. Las variaciones de la biomasa de macrófitos sumergidos no se ven afectadas por los cambios de salinidad, aunque sí existe un cambio considerable en la composición específica de las comunidades (tabla 2).

El patrón de variación de la biomasa de macrófitos con la profundidad (fig. 4) es similar al que, a una escala de profundidades mucho mayor, se observa en lagos de agua dulce (SPENCE, 1982). En este modelo lacustre, el perfil más o menos parabólico de la relación entre la biomasa y la profundidad se ha interpretado como el resultado, por un lado, del efecto de la extinción de la luz a profundidades elevadas y por otro, de la acción conjunta del efecto físico del oleaje y la limitación del espacio en profundidades más someras (DuARTE \& KALFF, 1988). La aparente limitación por la luz a que están sometidas las comunidades de macrófitos sumergidos de la marisma de Doñana, a pesar de su escasa profundidad (tabla 1), se interpreta como el resultado de la fácil resuspensión de arcillas y limos del sedimento por efectos mecánicos.

La acción del viento a través del oleaje explica la gran turbiedad durante el invierno (fondos desprovistos de vegetación sumergida) en las zonas más expuestas (lucios y caños) en contraposición a las más protegidas (quebradas, marisma a sotavento de las vetas, canales), donde se ve el fondo incluso a profundidades superiores a los $90 \mathrm{~cm}$.
No obstante, durante la primavera, la relación entre el área de exposición al oleaje y la transparencia del agua no es significativa ni de signo negativo $(r=0,18 ; n=53)$. Esto se debe al efecto estabilizador de las praderas de macrófitos acuáticos sumergidos sobre los materiales finos de las capas superiores del sedimento (Bulthius et al., 1984). De esta forma, considerables extensiones de agua libre de grandes lucios con tapices continuos de macrófitos sumergidos (por ejemplo, la zona $\mathrm{E}$ del lucio de los Ansares) mantienen, a pesar de estar sometidas a la acción de los vientos, aguas muy transparentes. Otros lucios, sin embargo, y algunos tramos de los caños tienen aguas más o menos turbias. Observaciones de campo sugieren una asociación entre el efecto desestabilizador de la pisada del ganado que se mueve por la marisma en busca de forraje y la turbiedad del agua y la ausencia de macrófitos sumergidos. En la figura 4, en la que a cada muestra se le ha asignado un símbolo según la intensidad del efecto de pisoteo del ganado, se observa que la tendencia parabólica de la distribución de puntos depende del grado de pisoteo. Las muestras con una intensidad de pisoteo media y alta poseen valores bajos o nulos de biomasa independientemente de su profundidad, mientras que la biomasa de estaciones poco o nada

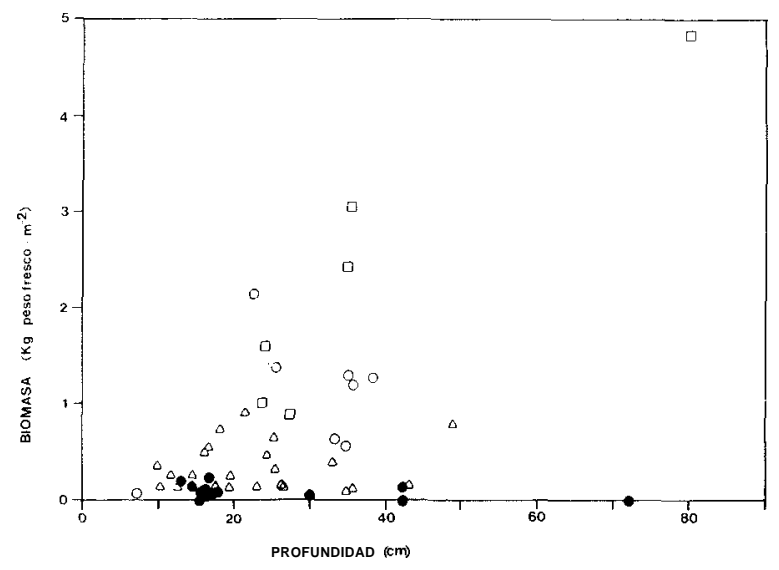

Figura 4.- Relación entre la biomasa de macrófitos acuáticos sumergidos y la profundidad del agua de la marisma. A cada muestra se le ha asignado un símbolo según la intensidad del efecto del ganado: $O$ nula; $O$ baja; $A$ media; 1 alta.

The relationship between submerged macrophyte biomass and water depth. Symbols represent $(O)$ null grazing pressure; $(O)$ low grazing pressure; $(A)$ medium grazing pressure; and $(O)$ high grazing pressure. 
afectadas por el ganado tienden a aumentar con la profundidad. Las muestras de los canales de los lucios de los Ansares y Mari López, con profundidad entre 56 y $80 \mathrm{~cm}$, registraron los valores más elevados de macrófitos sumergidos (3052-4844 g peso fresco $\mathrm{m}^{-2}$ ) y mantienen aguas muy transparentes. Esta tendencia a encontrar los valores máximos de biomasa asociados a zonas más profundas también se registra en otras marismas similares como las de Camargue, en el sur de Francia (Grillas \& Duncan, 1986).

La exposición del sustrato a los efectos del oleaje, resultante de la destrucción del tapiz vegetal por las pisadas del ganado, posibilita la resuspensión de los materiales finos en la columna de agua, de otra forma impedida por la cubierta de macrófitos, como indica la relación inversa $(r=-0,55$; $\mathbf{P} \leqslant 0,01, \mathbf{n}=50$ ) entre la transparencia del agua y la intensidad del pisoteo del ganado. Los datos obtenidos permiten postular la existencia de un valor crítico de la intensidad del pisoteo del ganado a partir del cual la turbiedad local generada conduce a la eliminación, por limitación de la luz, del tapiz sumergido adyacente; este proceso se propagaría según un mecanismo de retroalimentación positivo (mayor zona expuesta $\rightarrow$ mayor turbiedad $\rightarrow$ aumento de la zona expuesta), que conduciría a la completa desaparición del tapiz sumergido en el área comprendida dentro de los límites de difusión turbulenta definidos por las dimensiones físicas de la cubeta. Esta hipótesis, evidentemente, necesita ser comprobada con experimentos de campo. También se ha observado cómo las zonas donde pasta o ha pastado el ganado, son utilizadas preferentemente por bandos de flamencos (Phoenicopterus ruber roseus) para su alimentación. El mecanismo alimenticio de este ave (pisoteo y remoción, «treadings», CRAMP \& SIMONS, 1977; «Pietinement pivoté» Johnson, 1983) hace que estas áreas no sean colonizadas por tapices densos de macrófitos. El resultado final se traduce en amplias zonas de la marisma con aguas turbias, verdosas a finales de primavera (desarrollo del fitoplancton) y con una baja diversidad biológica (MonTEs \& Bernues, en prensa).

Otro factor biótico de gran importancia para la regulación de la cobertura de los macrófitos acuáticos de la marisma más dulce es el consumo de éstos por el cangrejo rojo americano (Procambarus clarkii Girard). Este crustáceo, introducido en las marismas del Guadalquivir en 1974, tiene limitada su reproducción a partir de aguas de carácter mixo-mesosalinas (LIBRERo, 1981). En la marisma alcanza densas poblaciones, muy activas, a lo largo del arroyo de la Rocina y en la parte central y septentrional del caño de las Madres (fig. 1). En estas zonas existían, antes de su introducción, extensos y densos tapices de macrófitos sumergidos y flotantes (BIGOT \& MARAZANOF, 1965), en particular poblaciones de Myriophyllum alterniflorum y Ranunculus baudotii, así como una importante fauna asociada. Las aguas de esta zona de la marisma se encuentran actualmente desprovistas del tapiz de vegetación acuática flotante y sumergida, a pesar de presentar aguas poco mineralizadas y relativamente profundas. El efecto negativo de los cangrejos sobre los tapices de macrófitos sumergidos $\mathrm{y}$, por tanto, sobre los procesos metabólicos de los ecosistemas acuáticos ha sido demostrado, entre otros, por LODGE \& LORMAN (1987) y FERMINELLA \& RESH (1989).

\section{CONCLUSIONES}

Los resultados presentados reflejan la gran importancia de los macrófitos acuáticos, especialmente los sumergidos y de una forma más localizada los flotantes, en el metabolismo de la marisma del Parque Nacional de Doñana. Adicionalmente, los tapices de macrófitos sumergidos y flotantes de la marisma modulan numerosas características físicas del medio (temperatura en profundidad, hidrodinamismo, extinción de la luz, substrato), químicas (balances de $\mathrm{O}_{2} \mathrm{y} \mathrm{CO}_{2}$, ciclos biogeoquímicos, $\mathrm{pH}$, nutrientes) y biológicas (organismos epífitos, herbívoros y detritívoros). Durante el período de máxima actividad de estos tapices emergentes, flotantes y sumergidos (febrero-mayo) la mayor parte de la productividad de la marisma de Doñana se basa en la producción de estos elementos y de sus algas epífitas asociadas.

Los resultados obtenidos indican que la cobertura de macrófitos constituye una comunidad muy frágil sujeta al efecto desestabilizador de perturbaciones externas que afectan al régimen lumínico a que están expuestos (por ejemplo, pisada del ganado, densos bandos de flamencos, cangrejos). Por ello, es fundamental que cualquier proyecto 
de gestión que pueda alterar de algún modo el ambiente lumínico de las aguas de la marisma, como el control del régimen de caudales y calidad de las aguas de entrada, se apoye en estudios científicos previos que permitan evaluar su impacto sobre la composición y estructura de las comunidades de macrófitos acuáticos.

\section{AGRADECIMIENTOS}

Evelyne nos animó y ayudó desinteresadamente en la toma de muestras y José M." Pérez de Ayala nos proporcionó dos días de muestreo mágicos. Blanca Ramos y Rafael Cadenas nos solucionaron muchos problemas logísticos. Pablo G. Murillo resolvió nuestras dudas taxonómicas. Este estudio ha sido financiado por un Convenio de Investigación entre el Instituto Nacional para la Conservación de la Naturaleza y el Departamento de Ecología de la Universidad Autónoma de Madrid.

\section{Bibliografía}

Allier, C. \& V. Bresset, 1978. Étude phytosociologique de la marisma et sa bordure. Carte phytosociologique. In: $D o-$ nana. Prospección e inventario de ecosistemas: 59-11. Monografias 18. ICONA.

Allier, C., V. Bresset, A. Lacoste \& F. González BerNÁLDEZ, 1978. La Marisma de la Reserve Biologique de Doñana (Espagne). In: Doñana. Prospección e inventario de ecosistemas: 111-129. Monografías 18. ICONA.

АMAT, J.A., 1980. Biología y ecología de la comunidad de patos del Parque Nacional de Doñana. Tesis Doctoral, Universidad de Sevilla.

Bernues, M. \& C. Montes (en prensa). Documentación bibliográfica para el estudio del Medio Natural del Parque Nacional de Doñana y su entorno. ICONA.

Bigot, L. \& F. Marazanof, 1965. Considerations sur l'écologie des invertebrés terrestres et aquatiques des Marismes du Guadalquivir (Andalucía). Vie Milieu 16(1): 441-473.

Bulthius, D.A., G.W. BRand \& M.C. Mobley, 1984. Suspensed sediments and nutnents in waters ebbing from seagrass-covered and denuded tidal mudflats in a southern Australian embayment. Aquat. Bot. 20: 257-266.

CANFIEld, D.E. \& M.V. Hoyer, 1988. Influence of nutrient enrichment and light availability on the abundance of aquatic macrophytes in Florida streams. Can. J. Fish. Aquatic. Sci. 45: 1467-1472.

CARPENTER, S.R. \& D.M. LODGE, 1986. Effects of submerged macrophytes on ecosystems processes. Aquat. Bot. $26(3-4): 341-370$
CRAMP, S. \& K.E.L. Simons (eds.), 1977. The birds of the Western Paleartic. Vol. I. Oxford University Press.

DaMONT, R. \& W. HARVEY, 1987. Experimental design. ANO$V A$ and regression. Harper \& Row Pub., New York, 508 pp.

DAvies-Colley, R.J., 1988. Measuring water clarity with a black disk. Limnol. Oceanogr. 33: 616-623.

DuARTE, C.M. \& J. KalfF, 1988. The influence of lake morphometry on the response of submerged macrophytes to sediment fertilization. Can. J. Fish. Aquat. Sci. 45: 216-221.

Duarte, C.M., J. KalfF \& R.H. Peters, 1986. Patterns in the biomass and cover of aquatic macrophytes in lakes. Can. J. Fish. Aquat. Sci. 43: 1900-1908.

FERMINELLA, J.N. \& V.H. RESH, 1989. Submerged macrophytes and grazing crayfish: an experimental study of herbivory in an California freshwater marsh. Holartic Ecology 12: 1-8.

GonzÁlez Bernáldez, F., L. Ramirez-Diaz, A. Torres \& F. Diaz PINEDA, 1977a. Estructura de la vegetación de la Marisma de la Reserva Biológica de Doñana (Huelva). I Análisis factorial de datos cualitativos. An. Edaf. Agrob. 36 (9-10): 989-1004.

González Bernaldez, F., L. Ramtrez Diaz, A. Torres \& F. Diaz PinedA, 1977b. Estructura de la vegetación de la Marisma de la Reserva Biológica de Doñana (Huelva). II Estudio de un gradiente de salinidad. An. Edaf. Agrob. 36 (9-10): 1005-1018

GoulDER, R. 1970. Day-time vanations in the rates of production by to natural communities of submerged macrophytes. J. Ecol. 58: 521-528.

Grillas, P. \& P. DunCan, 1986. On the distribution and abundance of submerged macrophytes in temporary marshes on the Camargue (S. France). Proc. EWRSIAAB 7th Symposium on Aquatic Weeds: 133-141.

HERNANDO, J.A. 1978. Estructura de la comunidad de peces de la Marisma del Guadalquivir. Tesis Doctoral, Universidad de Sevilla.

Johnson, A.R., 1983. Etho-Écologie du flamant rose (Phoenicopterus ruber roseus) en Camargue et dans Ouest paleartique. Thèse, Université Paul Sabatier, Toulouse.

JUPP, B.P. \& D.H.N. SPENCE, 1977. Limitations of macrophytes in a eutrophic, Loch Leven, II. Wave action, sediments and waterfowl grazing. J. Ecol. 65: 431-446.

Librero, M. 1981. Biología y pesca del Cangrejo. In: El Cangrejo rojo de la Marisma: 17-23. Junta de Andalucía.

Lind, C.T. \& G. CotTAM, 1969. The subrnerged aquatics of University Bay: A study in eutrophization. Am. Midl. Nat. 81: 353-369.

LodGe, D.M., \& J.G. LoRman, 1987. Reductions in submerged macrophyte biomass and species richness by the crayfish Orconectes rusticus. Can. J. Fish. Aquat. Sci. 44: 591-597.

Llamas, R., F.J. Rodriguez Arévalo, J. Tenajas \& A. Vela, 1987. El Parque Nacional de Dofiana: El Medio Físico. In: Bases Científicas para la protección de los Humedales españoles: 147-172. Real Academia de Ciencias de Madrid.

Montes, C., J.A. Amat \& L. Ramirez-Diaz, 1982a. Ecosistemas acuáticos del Bajo Guadalquivir (SW. España). I. Características generales físico-químicas y biológicas de las aguas. Studia Oecologica 3: 129-158.

Montes, C., L. Ramirez Diaz \& A.G. Soler, 1982b. Variación estaciona1 de las taxocenosis de Odonatos, Coleopteros y Heterópteros acuáticos en algunos ecosistemas del 
Bajo Guadalquivir (SW España) durante un ciclo anual. $A n$. Univ. Murcia 38 (1-4): 19-100.

Montes, C., J.A. Amat \& L.R. Diaz, 1982c. Distribución temporal de las caracteristicas físico-químicas y biológicas de las aguas de algunos ecosistemas del Bajo Guadalquivir (SW España) a lo largo de un ciclo anual. An. Univ. Murcia 38 (1-4): 209-304.

Montes, C. \& M. Bernues, en prensa. Incidencia del flamenco rosa (Phoenicopterus ruber roseus) en el funcionamiento de los ecosistemas acuáticos del Parque Nacional de Donana. Reunión Técnica sobre la situación y Problemática del Flamenco rosa en el Mediterráneo Occidenral y Africa Noroccidental. A.M.A. Andalucía.

Rivas Martínez, S., M. Costa, S. Castroviejo \& E. ValDÉs, 1980. Vegetación de Doñana (Huelva, España). Lazaroa 2: 5-189.
SPENCE, D.H.N. 1982. The zonation of plants in freshwater lakes. Adv. Ecol. Res. 12: 37-125.

SymoENS, J.J. (edit.) 1988. Vegetation of Inlands waters. Klumer Academic Publishers, 400 pp.

Tenajas, J. 1984. Contribución a la hidrogeoquímica de las marismas del Parque Nacional de Doñana con aplicación del análisis de imágenes Landsat. Tesis de Licenciatura. Universidad Complutense de Madrid. 240 pp.

Torres, A., L. Ramirez Diaz, F. Diaz Pineda \& F. GonZÁLEZ BERNÁLDEZ, 1978. Estructura y variación de la vegetación de la Marisma de la Reserva Biológica de Doñana. In: Doñana. Prospección e inventario de ecosistemas. 225-243. Monografías 18. ICONA.

WeTZEL, R.G., 1983. Limnology. 2a. ed. Saunder, Toronto.

WISTSLAKE, D.F., 1965. Theoretical aspects of the comparability of productivity data. Mem. Inst. Ital. Idrobiol. (suppl.): 313-322. 